\title{
Game of Thrones - Fernsehserien als Artikulation gesellschaftlich-unbewusster Phantasien
}

\author{
Gerald Poscheschnik
}

\begin{abstract}
Zusammenfassung
Kulturelle Produkte, wie z.B. Fernsehserien, lassen sich als Artikulationsformen gesellschaftspolitischer Phantasien verstehen, die bewusste und unbewusste Elemente in sich bergen. Game of Thrones, ein furioses Fantasy-Mittelalterspektakel, aufgeladen mit Gewalt und Sex, scheint auf den ersten Blick nur wenig tagesaktuelle Bezüge aufzuweisen. Eine psychoanalytische Untersuchung, die die Serie ihres manifesten Gewands entkleidet, legt wesentliche Elemente und ihre Relationen frei, die frappante Parallelen zu rezenten gesellschaftspolitischen Phantasien offenlegen. Die Verhältnisse am fiktiven Kontinent Westeros bieten sich als Zeitdiagnose einer niedergehenden Kultur an, deren Potentaten völlig von einem Ränkespiel um Macht und Einfluss absorbiert sind und ihre Wahrnehmung all dem verweigern, was nicht ihrem eigenen Machterhalt dient. Der durch eine Mauer abgeschottete Norden des Landes kann als Deponie für die verdrängten Nebenwirkungen des eigenen Treibens, wie z.B. Umweltzerstörung und menschliches Elend, verstanden werden, deren deletäre Wirkung letztlich die eigene Existenz bedroht. Der ferne Kontinent Essos repräsentiert die Hoffnung, es könnte noch eine Wende oder Alternative entstehen. Die Medienpädagogik kann einen bildungstheoretischen Beitrag zum Verständnis moderner Fernsehserien leisten, benötigt dazu aber auch den interdisziplinären Austausch.
\end{abstract}

Game of Thrones - TV Series as Articulations of Unconscious Sociopolitical Fantasies

\begin{abstract}
Cultural products such as films or tv-series can be understood as articulations of sociopolitical fantasies that contain conscious and unconscious elements. Game of Thrones, a furious medieval spectacle, full of violence and sex, prima facie seems to offer hardly any current references. A psychoanalytic examination, which disrobes the series from its manifest vesture, reveals essential elements and their relations that bear remarkable analogies to prevailing sociopolitical fantasies. The circumstances on the fictitious continent Westeros provide a diagnosis of a descending culture whose potentates are completely absorbed by intrigues
\end{abstract}


for power and influence. They refuse to notice anything that does not serve to retain their power. The North, cut off from Westeros by a tremendous wall, can be understood as a repository for the suppressed side effects of the own ado, such as ecological devastation or human hardship. Their effects threaten our own existence in the long run. The distant continent Essos stands for the hope for a turn toward an alternative world. Media pedagogy can contribute educational theories to the understanding of modern TV series, but needs an interdisciplinary dialogue for this purpose.

\section{Einleitung}

Grosse Popularität eines Films oder einer Fernsehserie ist vermutlich nicht nur der Darstellung klassisch-zeitloser Sujets geschuldet, sondern auch der expliziten oder impliziten Thematisierung von gesellschaftlich virulenten Umständen. Das zentrale Narrativ der Inszenierung funktioniert vor allem dann, wenn es den Nerv der Zeit trifft, also ein zeitgeistiges Thema repräsentiert. Stephen King (2012) hat in seiner theoretischen Auseinandersetzung mit dem Horrorgenre festgehalten, dass es im Verlauf der Historie immer wieder zu bestimmten realen gesellschaftlichen Ängsten, Schocks oder Traumata kommt, die bestimmte Horrorgeschichten auf fruchtbaren Boden fallen lassen. Auf einer bewussten Ebene operiert Horror, indem er sein Publikum schockt und/oder anwidert; auf einer unbewussten Ebene aber betätigen die entsprechenden Geschichten phobische Druckpunkte (phobic pressure points), die oft massenhaft verbreitete gesellschaftliche Ängste politischer und ökonomischer Natur aktivieren. Diese können sich durchaus von Dekade zu Dekade wandeln. Als Beispiel bringt King den Mitte der 50er-Jahre beginnenden Wettlauf ins Weltall zwischen den Supermächten USA und UdSSR und vor allem den Schock, der in der westlichen Welt ausgelöst wurde, als es der Sowjetunion überraschend schnell gelang 1957 den Sputnik-Satelliten in einen Orbit um die Erde zu schiessen. In dieser Zeit erschienen massenhaft Science-Fiction-Geschichten, die von einer glorifizierten Eroberung und Kolonisierung des Weltraums handelten; darunter gab es allerdings auch Sci-Fi-Horror-Geschichten, in denen eine Invasion und Zerstörung der Welt durch ausserirdische Mächte dargestellt wurde. Hierbei entsprachen die ausserirdischen Usurpatoren/innen symbolisch der kommunistischen Bedrohung, die Zerstörung des Planeten der atomaren Vernichtung. Auch die Psychoanalyse postuliert, dass nicht nur das Individuum, sondern auch eine Gesellschaft als Kollektiv Phantasien über sich selbst und ihren gegenwärtigen Zustand produziert, in denen u. a. Ängste und Hoffnungen konserviert sind. Diese Phantasien zeichnen sich durch einen bewussten Mantel aus, der einen unbewussten Kern verhüllt (Erdheim 1984; Fromm 2006). Kulturelle Produkte, seien es erfolgreiche Filme oder Fernsehshows, lassen sich als Artikulationsform solcher gesellschaftlichen Phantasien verstehen. 


\section{Game of Thrones als symbolischer Ausdruck einer gesellschaftspolitischen Phantasie}

Die vom amerikanischen Sender HBO produzierte Fernsehserie Game of Thrones (GoT, 2011-) begeistert international seit einigen Jahren ein Millionenpublikum und wird von der Kritik überwiegend gefeiert, ja gilt sogar als ein Kulminationspunkt des modernen Quality TV bzw. Complex TV (Mittell 2015). GoT ist eine Fernsehadaption der Fantasy-Romanreihe Das Lied von Eis und Feuervon George R. R. Martin, dem als historische Inspiration u. a. die Rosenkriege des 15. Jahrhunderts in England dienten, in denen die Yorks und die Lancasters um die Krone rivalisierten, und die schon von Shakespeare (z. B. Henry VI, Richard III) aufgegriffen wurden. GoT verwebt solche Fäden des klassischen Dramas mit Elementen von Trash, Splatter, Horror, Porno und Fantasy.

Im Internet kursieren diverse - durchaus stimmige - Versuche, die einzelnen Charaktere von GoT mit psychiatrischen Diagnosen zu versehen. Der bunte Reigen dieser Diagnosen bewegt sich von posttraumatischer Belastungsstörung über Borderline, Narzissmus und Alkoholismus bis hin zu Depressionen und antisozialen Persönlichkeiten. Meine psychoanalytische Interpretation von GoT geht auf das Schicksal der einzelnen Protagonisten/-innen lediglich exemplarisch und verkürzt ein und richtet sich stattdessen auf die Gesamtinszenierung, den dramatischen Kern der ganzen Serienhandlung und versteht diesen als symbolischen Ausdruck momentan virulenter gesellschaftlich-unbewusster Phantasien (für Details zur psychoanalytischen Methodik siehe Lorenzer 1988; Poscheschnik 2015).

Auf der manifesten Inhaltsebene ist GoT in einer historisch und topographisch fernen, ja sogar fiktiven Fantasy-Mittelalterwelt angesiedelt; die Serie besticht u.a. durch nackte und entblösste oder zerstückelte und verstümmelte Körper; sie ist ein Mittelalterspektakel mit übernatürlichen Kreaturen und einer Freakshow grausamster Machthaber/innen, die zwar schauspielerisch überzeugen können, aber offensichtlich überspitzt sind. Auf den ersten Blick bietet diese Fantasyorgie aus Sex, Blut, Gewalt, Drachen und Wiedergängern nur wenig Berührungspunkte und Parallelen zum privaten und gesellschaftlichen Leben eines/ $r$ durchschnittlichen, zeitgenössischen Zuschauers/-in. Auf der szenisch-unbewussten Ebene, der latenten Sinnebene, die sich offenbart, wenn man von der Serie die genretypischen Elemente subtrahiert und ihre Handlung auf die wesentlichen Elemente und Relationen reduziert, zeigt sich ein komplexes Drama, das das Empfinden vieler Menschen vom Status quo unserer heutigen westlichen Gesellschaft widerzuspiegeln scheint. Die Topographie von GoT mit ihren drei zentralen Orten (der Kontinent Westeros, der Norden, der Kontinent Essos) und den dortigen Geschehnissen lassen sich als Bausteine dieser mehr oder weniger vagen Phantasie über die gegenwärtige Verfasstheit unserer Gesellschaft lesen. So gesehen wird GoT zur audiovisuellen Repräsentation von gesamtgesellschaftlichen Dynamiken, die plötzlich 
alles andere als fremd wirken. In den folgenden Abschnitten werde ich versuchen zu zeigen, dass die kriegerischen Ereignisse auf Westeros das Gefühl vieler Menschen widerspiegeln, das gesellschaftliche Subjekt sei nur noch ohnmächtige/r Statist/in in einem macht- und geldpolitischen Spiel mit wenigen Gewinnern und vielen Verlierern. Danach gehe ich auf die Geschehnisse im fernen Norden ein, die die Angst und Ahnung vieler Menschen zum Ausdruck bringen, das vermeintlich alternativlose Noch-mehr-des-Gleichen wird über früher oder später mit dem völligen Kollaps des Systems bezahlt werden müssen. Die sich am Kontinent Essos zutragende Handlung schliesslich transportiert die vorsichtige Hoffnung besorgter Bürger/innen, es könnte vielleicht doch noch zu einem Wandel hin zum Besseren kommen.

\section{Westeros - Ränkespiel um den Eisernen Thron}

Der fiktive Kontinent Westeros, auf dem sich massgebliche Teile der Handlung abspielen, befindet sich zivilisatorisch und technologisch in etwa am Stand des Mittelalters und ist klimatisch von sehr langen, sich über Jahre erstreckenden Wintern und Sommern geprägt. Gegen Ende eines solchen Sommers wird der amtierende König Robert Baratheon, ein notorischer Säufer und Weiberer, auf der Jagd von einem Schwein letal verletzt, woraufhin ein Ränkespiel um den so genannten Eisernen Thron losgetreten wird. Einflussreiche Familiendynastien versuchen nun mit den Mitteln der Intrige, der Diplomatie, militärischer oder ökonomischer Macht ihren eigenen Anspruch geltend zu machen oder den von ihnen favorisierten Thronanwärter durchzusetzen. Die Mächtigen des Landes imponieren dabei nicht als Patrone/-innen mit edler Gesinnung und tadellosem Leumund, vielmehr handelt es sich überwiegend um geld- und machtgierige, moralisch in vielerlei Hinsicht verderbte und oft sadistische Machiavellisten/-innen. Joffrey Baratheon beispielsweise, der vermeintliche Sohn des verunfallten Königs, der ihm auf den Thron nachfolgt, ist eigentlich das Ergebnis von Untreue und Inzest seiner Mutter Cersei, der Witwe des Königs, mit ihrem eigenen Bruder. Seine eigene Verlobte, deren Vater er wegen vermeintlichen Verrats enthaupten liess, demütigt und misshandelt er öffentlich. Aber auch andere Aspiranten/-innen auf den Thron zeichnen sich durch Wahnsinn und Grausamkeit aus; Robert Baratheons Bruder Stannis wähnt sich als göttlich legitimierter Herrscher, der nicht nur politische Opponenten/-innen, sondern letztlich sogar seine eigene Tochter am Scheiterhaufen verbrennen lässt, in der vergeblichen Hoffnung, so seinen Gott günstig zu stimmen. Aber selbst die scheinbaren Lichtgestalten unter den Protagonisten/-innen, wie z. B. Robb Stark oder Jon Snow, strafen drakonisch und dekapitieren jene, die sich ihnen nicht beugen wollen. Wer in diesem Krieg nicht ständig auf der Hut ist, muss damit rechnen, dass Macht jäh in Ohnmacht umschlägt: Die Familie Stark, mächtige und loyale Verbündete des Königs, wird nach dessen Tod beinahe vollständig ausradiert. Der 
tyrannische König Geoffrey wird auf seiner eigenen Hochzeit vergiftet und krepiert elendiglich vor aller Augen. Dem gemeinen Fussvolk ist in diesem Ränkespiel übrigens nur eine marginale Rolle beschieden. Die einfachen Leute sind primär Spielfiguren für die herrschende Klasse in ihrem Kampf um die Suprematie; sie werden entweder als Opfer von Plünderungen marodierender Soldateska oder mehr oder weniger widerwillig Kriegsdienst Leistende, die verstümmelt oder getötet werden, dargestellt.

Die Vorgänge auf Westeros scheinen szenisch eine Phantasie einzufangen, die viele Menschen in unserer von einer globalen Wirtschaftskrise gebeutelten Gesellschaft teilen. Der Tod des Königs, der drohende Einbruch eines langen Winters und die Kulisse des Mittelalters, das vielleicht nicht ganz zu Unrecht im kulturellen Gedächtnis als finsteres Zeitalter gespeichert ist, vermitteln die äusserst zeitgemässe Stimmung, dass eine bestehende Ordnung untergeht und die Zeiten schlechter zu werden drohen. Der Kampf jeder gegen jeden und die dräuende Gefahr von Ohnmacht und Vernichtung spiegeln einen auch in unserer Kultur härter werdenden Kampf um Anerkennung (Honneth 2010) wider. Die verstetigte Wirtschaftskrise lässt den von den vorhergehenden Generationen erworbenen Wohlstand der ohnehin schrumpfenden Mittelschicht erodieren. Die Verschärfung des Konkurrenzkampfs zwischen Staaten, im Zuge dessen Arbeitsplätze nach Asien und Südamerika verlagert werden, verstärkt auch den innergesellschaftlichen Konkurrenzkampf und die Prekarisierung der Arbeitsverhältnisse. Europa bspw. stemmt sich nach Jahrzehnten des wirtschaftlichen Aufschwungs gegen seinen Niedergang, mitunter indem es seine sozialen Errungenschaften opfert. Die Menschen spüren, dass sie austauschbare Arbeitssklaven geworden sind, deren Job jederzeit abgebaut, anderswo hin verlagert oder einem/r anderen, motivierteren Kandidaten/ in gegeben werden kann (s. a. Beck 1986). In einer Gesellschaft, in der Wohlstand nur noch etwas ist, das sich auf Seiten der ohnehin schon Reichen und Betuchten mehrt (Piketty 2014), wird der soziale Abstieg für viele zur realen Bedrohung (s.a. Nachtwey 2016). Die archaische Brutalität, mit der in GoT gewütet wird, passt zum Gefühl, dass dieser Kampf für viele als existenziell bedrohlich und härter werdend erlebt wird. Völlig ohnmächtig muss der/die Einzelne ein Spiel spielen, dessen Regeln ihm/ihr von anderen aufoktroyiert werden. Dass die Mehrzahl der Zuschauer/ innen von GoT im reicheren Teil der Welt lebt, tut diesem subjektiven Erleben nur wenig Abbruch. Auch der Eindruck, den das Ränkespiel der regierenden Kaste von Westeros vermittelt, die Mächtigen würden nur noch sich selbst und ihrem eigenen Vorteil dienen, das Volk für ihre Zwecke instrumentalisieren und ausbeuten, scheint sich auch in westlichen Demokratien mehr und mehr zu verbreiten. Wirtschaftspolitische Weichenstellungen, wie z. B. Freihandelsabkommen, deren Impact gerade die breite Masse der Bevölkerung trifft, werden scheinbar ohne demokratische Kontrolle vorgenommen (siehe z. B. Crouch 2008; Streeck 2013). Von der desillusio- 
nierten Bevölkerung hört man dementsprechend immer öfter, dass der Kampf der politischen Eliten um die Macht nur noch dem eigenen Selbsterhalt dient; Parteien und Politiker unterschiedlicher Coleur scheinen sich kaum noch voneinander zu unterscheiden. Sie dienen nur noch sich selbst und vermeintlichen ökonomischen Zwängen, die als unausweichlich und alternativlos präsentiert werden.

\section{Der Norden - Hinter der Mauer lauert todbringendes Unheil}

Die Kronlande werden durch eine schon vor Äonen errichtete, kolossale Mauer vom eisigen, beinahe gänzlich vergletscherten Norden Westeros' abgeschirmt. Zur Bewachung der Mauer wurde die Nachtwache gegründet, schwarz gekleidete Männer, die ein Treuegelübde schwören, das ihnen unbedingte Loyalität ihrer Aufgabe gegenüber abverlangt, Ehe, Kinder, Familie verbietet und von dem sie erst mit ihrem Tod entbunden sind. Was nach hehrem Ethos klingt, ist tatsächlich ein zusammengewürfelter Trupp aus überwiegend Kriminellen, die sich der Nachtwache verpflichtet haben, um Kerker oder Todesstrafe zu entrinnen. Hinter der Mauer herrscht weitgehende Anarchie, die dort lebenden Menschen werden Wildlinge genannt. Staatliche Ordnung, zivilisatorische Errungenschaften wie Städtebau und die Submission unter eine Obrigkeit sind ihnen weitgehend fremd. Ein Beispiel für die völlige Tabulosigkeit jenseits der Mauer ist Craster, ein alter mürrischer Mann, der mit einer Schar seiner Töchter zusammenlebt, mit denen er ein inzestuöses Verhältnis pflegt. Die Töchter, die er mit diesen zeugt, behält er als angehende Geliebte, die Söhne tötet er noch als Neugeborene. Während in Westeros der Krieg um den Eisernen Thron wütet, spielen sich im Norden unheimliche Dinge ab. So genannte Weisse Wanderer, untote Kreaturen, die in alten Überlieferungen erwähnt, aber im heutigen Westeros lediglich für einen Mythos gehalten werden, werden plötzlich wieder gesichtet. Die sich zusammenbrauende Bedrohung, nötigt die Nachtwache schliesslich, die Regenten/-innen von Westeros zu warnen und um Unterstützung zu bitten. Angesichts des dräuenden Unheils, von einer Armee von Untoten überrannt und vernichtet zu werden, müsste das Ränkespiel um den eisernen Thron eigentlich zur Bedeutungslosigkeit verkommen. Die Warnungen vor der Gefahr werden von den Verantwortlichen allerdings in den Wind geschlagen.

Die völlige Selbstabsorption mit dem eigenen Ränkespiel und das geflissentliche Ignorieren der Gefahr lassen die Mauer und den Norden psychologisch betrachtet als Mise en Scène des aus der bewussten Wahrnehmung Ausgeschlossenen, des Verdrängt-Unbewussten, erscheinen. Die Symbolik der wiederauferstandenen Toten und ihr Impetus, die Mauer zu überwinden, verweist auf die unzähligen Leichen im Keller, die das wahnwitzige Treiben von Westeros erzeugt hat, und lässt sich als Wiederkehr des Verdrängten lesen (Freud 1915). Dinge und Kreaturen, die uns unheimlich erscheinen, sind freudianisch betrachtet zwar äusserlich fremd 
und unvertraut, sie tangieren allerdings einst vertraute und dann verdrängte Komplexe (Freud 1919). Auch der kapitalistische Modus vivendi der westlichen Gesellschaften erzeugt unschöne Abfallprodukte, deren Existenz für gewöhnlich der Verdrängung anheim fallen gelassen wird, die aber auf uns zurückzufallen drohen. Die Produktion der Güter, die tatsächlich oder vermeintlich für unser tägliches Leben gebraucht werden, erwirkt einen hemmungslosen Raubbau an den Ressourcen unseres Planeten und einen schonungslosen Umgang mit der Natur. Die Verschmutzung von Luft, Boden und Wasser, Rodungen, Desertifikation oder der Treibhauseffekt zerstören zwar nicht kurzfristig, aber à la longue die Umwelt als Lebensgrundlage aller Menschen. Auf der Schattenseite der Globalisierung und des enthemmten Kapitalismus tummeln sich zudem unzählige menschliche Opfer. Die Ausbeutung ärmerer Länder ist eine Grundlage für hiesigen Wohlstand. Direkte oder indirekte militärische Interventionen des Westens in Konfliktherde ferner Länder sind mitverantwortlich fürs Elend der dortigen Zivilbevölkerung und Flüchtlingsströme. Aber auch in unserer eigenen Gesellschaft kommt es trotz Wirtschaftswachstums zu Massenarbeitslosigkeit und einer Verarmung immer breiterer Bevölkerungsschichten. Die Jugendarbeitslosigkeit bspw. hat selbst in vielen wohlhabenden Ländern Europas schwindelerregende Höhen erreicht. Wenn ich diese und ähnliche Themen als gesellschaftlich verdrängt betrachte, will ich damit keinesfalls sagen, sie würden niemals öffentlich diskutiert werden. Verdrängung ist laut Freud (1915) auch keine statische Kategorie, sondern ein dynamischer Prozess. Selbstverständlich wird z. B. über den Treibhauseffekt oder die Jugendarbeitslosigkeit sporadisch berichtet, allerdings sind diese Themen - ganz im Widerspruch zu ihrer tatsächlichen Brisanz - eben keine Dauerbrenner; sie blitzen medial immer wieder kurz auf, werden dann aber lieber möglichst schnell wieder «vergessen». Klimaprotokolle bspw. werden zwar immer wieder ratifiziert, aber letztlich so weit in die Zukunft verschoben, dass die Massnahmen stets getrost der nächsten Generation von Politikern überlassen werden können; ausgehöhlt und unterminiert werden die Vereinbarungen zusätzlich noch durch den Emissionsrechtehandel. Fazit bleibt, auch unsere eigene Zivilisation ist in gewisser Weise bedroht von selbst erzeugten Problemen, die zwar beharrlich verdrängt werden, nichtsdestotrotz aber drohen irgendwann wie ein Damoklesschwert auf uns niederzufahren.

\section{Essos - Hoffnung auf eine andere Welt}

Im weit entfernten, durch ein Meer von Westeros getrennten Kontinent Essos leben die letzten Nachfahren/-innen des vormaligen Herrschergeschlechts von Westeros, die Targaryens, im Exil. Bevor diese vom verstorbenen König Robert Baratheon gestürzt wurden, bauten sie ihre Macht auf der Kontrolle mittlerweile ausgestorbener feuerspeiender Drachen auf. Um an eine Armee zu gelangen und den Eisernen Thron zurückzuerobern, verheiratet Viserys seine Schwester Daenerys 
Targaryen noch als Jugendliche mit dem mächtigen Stammesführer Khal Drogo. Nach dem Tod ihres Bruders und ihres Gatten begibt sie sich mit drei vermeintlich versteinerten Dracheneiern in jenes Feuer, das den Leichnam ihres verstorbenen Mannes einäschern soll; sie überlebt nicht nur völlig unverletzt, sondern aus den Eiern schlüpfen auch drei kleine Drachen. Allmählich gelingt es ihr, sich zu neuer Macht emporzuschwingen. Sie baut eine Armee auf und befreit mehrere Städte in Essos von der Sklaverei. Obgleich Daenerys in ihren Entscheidungen oft wankelmütig wirkt und durchaus nach dem Talionsgesetz richtet, zeichnet sie sich durch Einsicht aus, dass es ihr noch an der nötigen Weisheit für eine gute Regentschaft mangelt. Mithilfe ihrer Berater übt sie sich deshalb als Herrscherin in den befreiten Städten, bevor sie nach Westeros übersetzen und den Eisernen Thron für sich beanspruchen will. Gerade mit dieser Einsichtsfähigkeit in die eigenen Grenzen scheint sie noch am ehesten die Züge einer Regentin zu tragen, die Westeros wieder Frieden und Gerechtigkeit bringen könnte.

Während Westeros vom Game of thrones völlig gebannt ist und man die verheerende Gefahr aus dem Norden bestenfalls vage ahnt, zeichnet sich im fernen Essos mit der Figur der Daenerys Targaryen ein Hoffnungsschimmer ab. Allerdings ist sie eine ambivalent gezeichnete Hoffnungsträgerin voller Unsicherheiten, die lediglich ein noch zu verwirklichendes Potenzial in sich birgt, zur weisen und gütigen Retterin in der Not zu werden. Im Unterschied zu anderen Charakteren ist sie aber immerhin bemüht, zu lernen und sich selbst als Regentin zu verbessern. Mit ihren Drachen scheint sie zudem auch über ein Rezept zu verfügen, das gegen die dunkle Bedrohung, die von jenseits der Mauer kommt, wirken könnte. Daenerys Targaryen repräsentiert als Regentin auf der Suche nach ihrer Identität somit kein fix und fertig ausgearbeitetes Konzept einer besseren Welt, sondern eine Hoffnung in statu nascendi. Gerade deshalb scheint sie treffsicher das dumpfe Gefühl vieler Menschen in unserer Gesellschaft zu verkörpern, dass zwar etwas falsch läuft, aber nicht so recht weiss, wie es anders gehen könnte. Einerseits existiert wohl eine relativ weit verbreitete Unzufriedenheit mit der aktuellen gesellschaftlichen Entwicklung und ein Wunsch nach Veränderung, gleichzeitig herrscht aber - nicht zuletzt durch das ideologische Vakuum, das durch den Untergang linker Utopien zurückgeblieben ist (Honneth 2015) - eine beinahe völlige Ahnungs- und Ideenlosigkeit, wie eine überzeugende Alternative aussehen könnte. Die von den Potentaten/innen gebetsmühlenartig asserierte Alternativlosigkeit des enthemmten Kapitalismus, die entgegen dem Offensichtlichen als einzige Problemlösungsstrategie die Parole «Noch mehr Kapitalismus» ausgibt, weckt Skepsis und nährt die Sehnsucht nach und Hoffnung auf eine andere, menschlichere Welt. Die Hoffnung als Gefühl ist also vorhanden, wie die sie realisierende Vision aussehen könnte, bleibt aber noch fraglich. Das Sprichwort sagt, die Hoffnung stirbt zuletzt; wenn man Game of Thrones trauen darf, muss sie sich überhaupt erst entwickeln und eine klare Gestalt annehmen. 


\section{Eine medienpädagogische Reflexion zum Abschluss}

Viele moderne Fernsehserien, darunter auch GoT, sind bestens dazu geeignet, die Zuseher/innen nicht nur zu unterhalten, sondern auch zu verstören. Fernsehserien, wie z. B. auch Breaking Bad (AMC 2008-2013) oder The Sopranos (HBO 1999-2007), um nur einige von vielen möglichen Beispielen zu nennen, brechen gezielt Erwartungshaltungen, die vom durchschnittlichen Zuseher/der Zuseherin an mediale Unterhaltung herangetragen werden. Auf Schwarz-Weiss-Zeichnungen der Charaktere wird beispielsweise konsequent verzichtet, weder gibt es wirklich Gute, noch völlig Böse, beinahe alle Figuren sind ambivalent gezeichnet und überraschend facettenreich konstruiert. Es gibt auch keine eindeutig identifizierbare Moral von der Geschicht', die den Versuch unternehmen würde, den Zuseher auf relativ plumpe Art und Weise zu erziehen. Weder obsiegen stets die Untadeligen, noch unterliegen immer die moralischen Tabubrecher/innen. Vielmehr werden Sympathieträger/innen, mit denen man mitfiebert und denen normalerweise ein viele Staffeln währendes Leben beschert wäre, jäh dahingemetzelt; ganze Handlungsstränge, die ein Potenzial zum Happy End in sich bergen würden, werden mit einem Streich ausgelöscht. Aber auch Rollen, wie der schon erwähnte Joffrey Baratheon, deren verächtliches Agieren vom Gros der Seher/innen vermutlich intuitiv mit Ressentiment bedacht wird, müssen stets damit rechnen, relativ schnell und unvermittelt das Zeitliche zu segnen.

Damit weigert sich GoT in vielerlei Hinsicht hartnäckig, sich einem einfachen Interpretationsschema zu fügen. Im Unterschied zu passiver Berieselung mit seichter Unterhaltung, die selbstverständlich auch ihre Berechtigung und ihren Nutzen hat, entzieht sich GoT - wie gewiss auch andere Quality-TV-Serien - mit seinen immer neuen Attacken auf eingeschliffene mentale Interpretationsschablonen einer simplifizierenden und vor allem quasi automatisch ablaufenden Deutung. Ich zweifle zwar nicht an einem kitschigen Happy End als Finale der letzten Staffel, allerdings lässt die bisherige Erzähldynamik einstweilen keinerlei vereinfacht moralisierende Interpretation zu. Ganz im Gegenteil wird der/die Zuschauer/in durch die Irritation, die die Handlung immer wieder erzeugt, auf sich selbst zurückgeworfen und mehr oder weniger gezwungen, sich allein und im Dialog mit anderen Zusehern/-innen einen Reim auf die Geschehnisse zu machen.

Genau hier kann die Medienpädagogik einen bildungstheoretischen Diskurs zum Verständnis audiovisueller Medien beitragen. Ich möchte mir erlauben, Überlegungen aus der Bildungstheorie von Koller (2011) als medienpädagogischen Zugang zu dieser Art des Fernsehens auszuborgen. Bildung, verstanden als Transformationsprozess der Figuren des Selbst- und Weltverständnisses durch krisenhafte Erfahrungen, kann nämlich auch in einem virtuellen Kontext passieren. Der Psychoanalytiker D.W. Winnicott (2015) hat hervorgehoben, dass Entwicklungsprozesse oft mit so genannten Übergangsobjekten bzw. in Übergangsräumen passie- 
ren. Das sind Objekte und Zonen, die einerseits zwar zur realen externen Umwelt gehören, zugleich aber mit subjektiver, psychischer Bedeutung aufgeladen sind und somit zwischen äusserlich-materieller und innerlich-psychischer Welt liegen. Fernsehen kann so gesehen zu einem Übergangsraum werden, in dem sich durchaus Bildungs- und Entwicklungsprozesse der ihn nutzenden Zuschauer/innen abspielen. Versteckt hinter dem furiosen Mittelaltergemetzel in GoT verbirgt sich ein Handlungskern mit verblüffend aktuellen gesellschaftspolitischen Referenzen, der den/die Zuseher/in zu kritischen Denk- und Reflexionsprozessen über sich selbst als politisches und gesellschaftliches Wesen sowie die Gesellschaft an sich animieren kann. Diese Prozesse passieren zwar zum einen Teil unbewusst, zum anderen Teil ist der/die Zuseher/in aber sehr wohl stark herausgefordert, Bedeutung aktiv und bewusst durch individuelle Denk- und interindividuelle Kommunikationsprozesse zu konstruieren. Insofern scheint es mir berechtigt, auch das Anschauen von Fernsehserien wie GoT als Bildungsprozess sui generis zu begreifen.

Die Medienpädagogik kann den wissenschaftlichen Diskurs über moderne Fernsehserien um einen solchen bildungstheoretischen Zugang bereichern. Voraussetzung dafür ist freilich eine Befreiung von ausschliesslich erziehungstheoretischem Denken und noch immer häufig kolportierten Klischees, die audiovisuelle Medien in Bausch und Bogen abkanzeln. Dazu zählen für mich auch die breit rezipierten Überlegungen von Horkheimer und Adorno (1988) zur von ihnen so bezeichneten Kulturindustrie, deren meist filmische Produkte den Zuseher/die Zuseherin zum reinen Konsumenten / zur Konsumententin degradieren und durch ihren manipulativen Gestus zur geistigen Stagnation verdammen würden. Dieser eigentlich frappierend undialektischen Betrachtung von Kultur als Ware zum Zwecke der Manipulation der Massen widersprechen deshalb auch dezidiert die Cultural Studies, welche die Dichotomie von Hochkultur und Populärkultur aufgebrochen haben und letztere als Gegenstand des Interesses ernst nehmen. Sie betonen nicht nur, dass im Populärkulturellen durchaus ein Moment der Subversion enthalten sein kann, sondern auch die aktive Rolle des Zuschauers / der Zuschauerin in der Konstruktion von Bedeutung (Marchart 2008; Winter 2010). Eine Medienpädagogik, die die subjektiven Reaktionen auf beliebte Fernsehserien als Rezeptionsleistung respektiert, kann vermutlich sogar adoleszente Schüler/innen am Sprung zum Erwachsenenalter erreichen. Kassandrahafte Warnungen von Lehrern/-innen, die Medienkonsum als Eskapismus deuten oder Serien wie GoT aufgrund eines gar zu oberflächlichen Blicks als gewaltverherrlichend kritisieren, sind dazu nur wenig geeignet. Wenn die Agents of Socialization (z. B. Lehrer/innen, Eltern) den Jugendlichen auf Augenhöhe begegnen, lassen sich Prozesse der Medienbildung in Gang setzen. Jörissens und Marotzkis (2009) Überlegungen zu den vier Dimensionen der Medienbildung (Wissensbezug, Handlungsbezug, Grenzbezug, Biographiebezug) könnten - evtl. 
erweitert um gesellschaftliche, historische und politische Akzente - hierfür dienlich sein.

Da kulturelle Phänomene aber nicht nur auf der bewusst-manifesten Inhaltsebene, sondern auch einer unbewusst-latenten Strukturebene ihre Wirkung auf die Zuseher/innen entfalten, kann der bildungstheoretische Blick der Medienpädagogik auch von der Psychoanalyse profitieren (Kutter u. Müller 2008; Laszig 2013; Žižek et al. 2002). Eine Analyse von blossen Inhalten, die auf der Oberfläche der Handlung verbleibt, ist oft zu wenig aussagekräftig, um verstehen zu können, was den psychischen Resonanzkörper des Publikums zum Schwingen bringt, zu Identifikationen und Kontraidentifikationen mit den Figuren einlädt und den emotionalen Sog bildet, keine Fortsetzung zu verpassen. Die psychoanalytische Methodik vermag unbewusste Gründe für die Faszination, die gewisse Filme und Serien auf ihre Zuschauer/innen ausüben, zu dechiffrieren. Die Offenlegung der latenten Sinnstruktur von medial transportierten Narrativen und Inszenierungen und der damit verbundenen szenisch-emotionalen Tiefenwirkung auf die Rezipienten/-innen ermöglicht ein Verstehen dieses medial transportierten Übergangs- und Reflexionsraums und damit auch der implizierten Bildungsmöglichkeiten.

\section{Literatur}

Beck, Ulrich. 1986. Risikogesellschaft: Auf dem Weg in eine andere Moderne. Frankfurt/Main: Suhrkamp.

Crouch, Colin. 2008. Postdemokratie. Frankfurt/Main: Suhrkamp.

Erdheim, Mario. 1984. Die gesellschaftliche Produktion von Unbewusstheit: Eine Einführung in den ethnopsychoanalytischen Prozess. Frankfurt/Main: Suhrkamp.

Freud, Sigmund. (1915) 1980. "Die Verdrängung.» In Gesammelte Werke. Bd. 10, hrsg. v. Alexander Mitscherlich, Angela Richards u. James Strachey, 247-261.

Freud, Sigmund. (1919) 1982. «Das Unheimliche.» In Studienausgabe. Bd. 4, hrsg. v. Alexander Mitscherlich u a., 241-274. Frankfurt/Main: Fischer.

Freud, Sigmund. (1921) 1974. "Massenpsychologie und Ich-Analyse.»In Studienausgabe. Bd. 9, hrsg. v. Alexander Mitscherlich u. a., 61-134. Frankfurt/Main: Fischer.

Fromm, Erich. 2006. Jenseits der Illusionen: Die Bedeutung von Marx und Freud. München: dtv.

Honneth, Axel. 2010. Kampf um Anerkennung: Zur moralischen Grammatik sozialer Konflikte. Frankfurt/Main: Suhrkamp.

Honneth, Axel. 2015. Die Idee des Sozialismus. Berlin: Suhrkamp.

Horkheimer, Max und Theodor Wiesengrund Adorno. 1988. Dialektik der Aufklärung. Philosophische Fragmente. Frankfurt/Main: Fischer. 
Jörissen, Benjamin und Winfried Marotzki. 2009. Medienbildung - Eine Einführung. Bad Heilbrunn: Julius Klinkhardt.

King, Stephen. 2012. Danse Macabre. London: Hodder \& Stoughton.

Koller, Hans-Christoph. 2011. Bildung anders denken: Einführung in die Theorie transformatorischer Bildungsprozesse. Stuttgart: Kohlhammer.

Kutter, Peter und Thomas Müller. 2008. Psychoanalyse: Eine Einführung in die Psychologie unbewusster Prozesse. Stuttgart: Klett-Cotta.

Laszig, Parfen. Hrsg. 2013. Blade Runner, Matrix und Avatare: Psychoanalytische Betrachtungen virtueller Wesen und Welten im Film. Berlin: Springer.

Lorenzer, Alfred. 1988. Kultur-Analysen: Psychoanalytische Studien zur Kultur. Frankfurt/Main: Fischer.

Marchart, Oliver. 2008. Cultural Studies. Konstanz: UVK.

Mittell, Jason. 2015. Complex TV: The Poetics of Contemporary Television Storytelling. New York u. London: New York University Press.

Nachtwey, Oliver. 2016. Die Abstiegsgesellschaft: Über das Aufbegehren in der regressiven Moderne. Berlin: Suhrkamp.

Piketty, Thomas. 2014. Das Kapital im 21. Jahrhundert. München: Beck.

Poscheschnik, Gerald. 2015. "Qualitative Auswertungsmethoden.»In Empirisch forschen: Die Planung und Umsetzung von Projekten im Studium, hrsg. v. Theo Hug u. Gerald Poscheschnik, 152-164. UTB 3357. 2. Aufl. Konstanz: UVK/Huter \& Roth.

Streeck, Wolfgang. 2013. Gekaufte Zeit: Die vertagte Krise des demokratischen Kapitalismus. Berlin: Suhrkamp.

Winnicott, Donald Woods. 2015. Vom Spiel zur Kreativität. Stuttgart: Klett-Cotta.

Winter, Rainer. 2010. Der produktive Zuschauer: Medienaneignung als kultureller und ästhetischer Prozess. Köln: Halem.

Žižek, Slavoj, Mladen Dolar, Alenka Zupancic, Stojan Pelko, Miran Bozovic und Renata Salecl. 2002. Was Sie immer schon über Lacan wissen wollten und Hitchcock nie zu fragen wagten. Frankfurt/Main: Suhrkamp. 